\title{
Electromagnetic waves in a Josephson junction in a thin film
}

\author{
R.G. Mints \\ School of Physics and Astronomy, \\ Raymond and Beverly Sacler Faculty of Exact Sciences, \\ Tel Aviv University, \\ Tel Aviv 69978, Israel \\ I.B. Snapiro \\ Physics Department, \\ Technion-Israel Institute of Technology, \\ Haifa 32000, Israel
}

\begin{abstract}
We consider a one-dimensional Josephson junction in a superconducting film with the thickness that is much less than the London penetration depth. We treat an electromagnetic wave propagating along this tunnel contact. We show that the electrodynamics of a Josephson junction in a thin film is nonlocal if the wave length is less than the Pearl penetration depth. We find the integro-differential equation determining the phase difference between the two superconductors forming the tunnel contact. We use this equation to calculate the dispersion relation for an electromagnetic wave propagating along the Josephson junction. We find that the frequency of this wave is proportional to the square root of the wave vector if the wave length is less than the Pearl penetration depth.
\end{abstract}

74.50.+r, 74.76. Db

Typeset using REVTEX 


\section{INTRODUCTION}

The electromagnetic properties of tunnel Josephson junctions are a subject of intensive studies over the past three decadest. A considerable attention is, in particular, attracted to the investigation of the SIS-type Josephson contacts. In this case the tunnel junction is formed by a thin layer of an insulator. This dielectric layer between two superconducting plates can be treated as a transmission line or a parallel plate resonator when the electromagnetic properties are concerned. It follows from this approach that an electromagnetic wave with a specific dispersion relation may propagate along the SIS-type Josephson junction2.

The existence of this Swihart electromagnetic wave results, in particular, in the self-

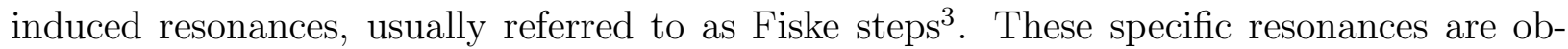
served as peaks in the current-voltage curves of the tunnel Josephson junctions. The study of Fiske steps is one of the methods to treat the electromagnetic properties of the SIS-type Josephson contacts. Recently, it was successfully applied to investigate the electromagnetic properties of the grain boundaries in $\mathrm{YBa}_{2} \mathrm{Cu}_{3} \mathrm{O}_{7-\delta}$ high-temperature superconductors 1 .

The self-induced Fiske resonances are observed in the presence of an external magnetic field, $H_{e}$. In this case the phase difference between the two superconductors forming the tunnel contact, $\varphi$, is a sum of two terms, i.e., $\varphi=\varphi_{H}+\varphi_{V}$. The first term, $\varphi_{H}$, describes

the increase of $\varphi$ along the Josephson junction (y-axis). The value of $\varphi_{H}$ is proportional to the magnetic field $H_{e}$ and is given by the formula $\varphi_{H}=-4 \pi \lambda H_{e} y / \Phi_{0}$, where $\lambda$ is the London penetration depth and $\Phi_{0}$ is the flux quantum. The second term, $\varphi_{V}$, represents the increase of $\varphi$ in time. The value of $\varphi_{V}$ is proportional to the voltage, $V$, applied to the contact, and is given by the formula $2 \mathrm{eVt} / \hbar$. As a result the tunnel current density

$$
j=j_{c} \sin \varphi=j_{c} \sin \left(\frac{2 e V}{\hbar} t-\frac{4 \pi \lambda H_{e}}{\Phi_{0}} y\right)
$$

is described by a wave propagating along the Josephson junction . The amplitude of this wave is given by the Josephson critical current density, $j_{c}$.

The self-induced Fiske resonances arise when the frequency of the tunnel current density wave, $2 \mathrm{eV} / \hbar$, becomes equal to the frequency of the Swihart electromagnetic wave 5 . 
Thus, the dependence of the frequency, $\omega$, on the wave vector, $k$, for an electromagnetic wave propagating along the Josephson junction determines the positions of the peaks in the current-voltage curve of a tunnel contact.

Usually, the dispersion relation $\omega(k)$ is determined by the sin-Gordon equation that leads $\operatorname{tol}$

$$
\omega=\omega_{j} \sqrt{1+k^{2} \lambda_{J}^{2}}
$$

where

$$
\omega_{j}=\sqrt{\frac{2 e j_{c}}{\hbar C}}
$$

is the Josephson frequency, $\mathrm{C}$ is the specific capacitance of the tunnel junction, and

$$
\lambda_{j}=\sqrt{\frac{c \Phi_{0}}{16 \pi^{2} \lambda j_{c}}},
$$

is the Josephson penetration depth.

The Swihart electromagnetic wave corresponds to the limiting case $1 \ll k \lambda_{J}$. It follows than from Eq. (2) that $\omega \approx \omega_{J} \lambda_{J} k$, i.e., this wave is propagating along the Josephson junction with a constant velocity

$$
c_{s}=\omega_{J} \lambda_{J}=\frac{c}{\sqrt{8 \pi \lambda C}}
$$

The linear dispersion relation $\omega=c_{s} k$ results in an equidistant set of peaks in the current-voltage curves of the SIS-type Josephson tunnel junctions 5 .

We can determine the phase difference $\varphi(y, t)$ in the mainframe of the local Josephson electrodynamics, i.e., by the sin-Gordon equation, as long as $k \lambda \ll 1$. It means, in particular, that for an electromagnetic wave propagating along the Josephson junction with $\lambda \ll \lambda_{J}$ the dispersion relation $\omega=c_{s} k$ is valid in the region $\lambda_{J}^{-1} \ll k \ll \lambda^{-1}$.

Let us now discuss the general case, i.e., the case when the restrictions on the wave vector $k$ are given by the inequalities $k d_{0} \ll 1$ and $k \xi \ll 1$, where $d_{0}$ is the thickness of the insulating barrier and $\xi$ is the coherence length. We treat here an SIS-type Josephson 
junction formed by two superconducting plates. In this case the space distribution of $\varphi$ is one-dimensional and the relation between the phase difference $\varphi(y, t)$ and the magnetic field in the superconductors is nonlocal if $1 \ll k \lambda$. As a result the function $\varphi(y, t)$ is determined by an integro-differential equation $\mathbf{3}$, i.e., the electrodynamics of a Josephson junction is nonlocal as far as the region of wave vectors $1 \ll k \lambda$ is concerned. Using this equation it was shown 910 that the dispersion relation for an electromagnetic wave with $1 \ll k \lambda$ takes the form

$$
\omega=c_{s} \sqrt{\frac{k}{\lambda}} .
$$

The phase velocity of this electromagnetic wave is inversely proportional to the square root of the wave vector $k$. It results in a non-equidistant set of the values of the voltage $V$ corresponding to the self-induced resonances in the current-voltage curve.

This effect is most pronounced when considering an SIS-type Josephson junction in a thin superconducting film with the thickness $d \ll \lambda$. In this case the space scale of magnetic field variation in the superconductors forming the contact is given by the Pearl penetration $\operatorname{depth} 11$

$$
\lambda_{\text {eff }}=\frac{\lambda^{2}}{d} \gg \lambda .
$$

Thus, the electrodynamics of a Josephson junction in a thin film is nonlocal if $1 \ll k \lambda_{\text {eff }}$. This region of wave vectors is much wider than the one given by the inequality $1 \ll k \lambda$.

In this paper we consider an infinite one-dimensional Josephson tunnel junction in a superconducting film with the thickness $d \ll \lambda$. We show that the electrodynamics of a Josephson contact is nonlocal if the space scale of variation of $\varphi$ is less than the Pearl penetration depth, i.e., if $1 \ll k \lambda_{\text {eff }}$. We derive the integro-differential equation determining the phase difference $\varphi(y, t)$. We use this equation to calculate the dispersion relation $\omega(k)$ for an electromagnetic wave propagating along an SIS-type Josephson junction.

\footnotetext{
${ }^{1}$ We present the theory of the self-induced Fiske resonances in the mainframe of the nonlocal Josephson electrodynamics elsewhere.
} 
The paper is organized in the following way. In Sec. II, we consider the electrodynamics of a long one-dimensional Josephson junction in a superconducting film with the thickness that is much less than the London penetration depth. We treat the general case of an arbitrary relation between the Josephson penetration depth and the effective Pearl penetration depth. We derive the integro-differential equation determining the phase difference distribution along the Josephson junction. In Sec. III, we apply this equation to calculate the dispersion relation for an electromagnetic wave propagating along the Josephson junction. In Sec. IV, we summarize the overall conclusions.

\section{BASIC EQUATIONS}

Let us consider a thin superconducting film ( $x y$-plane) with an SIS-type Josephson junction along the $y$-axis as it is shown in Fig. 1. We treat here the case when $\lambda \gg \xi$, in which the London equations govern the fields and currents inside the superconductor. Thus, outside the tunnel contact the relation between the current density $\mathbf{j}$ and the magnetic field $\mathbf{h}$ is given by 2

$$
\mathbf{h}+\frac{4 \pi \lambda^{2}}{c} \operatorname{rot} \mathbf{j}=0
$$

Introducing the vector potential A and combining Eq. (8) with the equation

$$
\mathbf{h}=\operatorname{rot} \mathbf{A}
$$

we express the current density $\mathbf{j}$ in the form

$$
\mathbf{j}=\frac{c}{4 \pi \lambda^{2}}(\mathbf{S}-\mathbf{A})
$$

where outside the tunnel junction the vector field $\mathbf{S}$ is given by the formula

$$
\mathbf{S}=\frac{\Phi_{0}}{2 \pi} \nabla \theta
$$

and $\theta$ is the phase of the order parameter. 
The quantities $\mathbf{j}, \mathbf{A}$ and $\mathbf{S}$ are nearly independent on the $z$ coordinate in the limiting case of a thin film, i.e., for $d \ll \lambda$. Therefore, in order to find the fields and currents we replace the superconducting film with the thickness $d \ll \lambda$ by an infinitely thin current-carrying sheet in the plane $z=0$. The current density $\mathbf{j}$ in this plane is determined then by the averaging of Eq. (10) over the thickness d11 3 , which results in

$$
\mathbf{j}=\frac{c}{4 \pi \lambda_{\text {eff }}}(\mathbf{S}-\mathbf{A}) \delta(z) .
$$

Let us now choose the London gauge, i.e., let us assume that $\operatorname{div} \mathbf{A}=0$. Then, substituting Eq. (12) into the Maxwell equation

$$
\operatorname{rot} \mathbf{h}=\frac{4 \pi}{c} \mathbf{j}
$$

we find the equation describing the vector potential $\mathbf{A}$ in the form

$$
-\Delta \mathbf{A}+\lambda_{\text {eff }}^{-1} \mathbf{A} \delta(z)=\lambda_{\text {eff }}^{-1} \mathbf{S} \delta(z)
$$

The vector field $\mathbf{S}$ is related to the phase difference

$$
\varphi(y)=\theta(+0, y)-\theta(-0, y) .
$$

This relation is given by the equation

$$
\operatorname{rot} \mathbf{S}=\frac{\Phi_{0}}{2 \pi} \varphi^{\prime}(y) \delta(x) \hat{\mathbf{z}}
$$

following from Eq. (11) and taking into account the singularity of the function $\theta(x, y)$ at $x=0$. The vector $\hat{\mathbf{z}}$ is here for the unit vector along the $z$-axis.

Applying the continuity equation $\operatorname{div} \mathbf{j}=0$ to Eq. (12) we find that $\operatorname{div} \mathbf{S}=0$. Thus, we can present the vector field $\mathbf{S}$ as a curl of a certain vector field $\mathbf{F}$, namely,

$$
\mathbf{S}(\boldsymbol{\rho})=\operatorname{rot} \mathbf{F}
$$

where $\boldsymbol{\rho}=(x, y)$ and

$$
\mathbf{F}=F(\boldsymbol{\rho}) \hat{\mathbf{z}}
$$


Substituting Eq. (18) into Eq. (16) we find the equation describing the function $F(\boldsymbol{\rho})$ in the form

$$
\Delta F=-\frac{\Phi_{0}}{2 \pi} \varphi^{\prime}(y) \delta(x)
$$

Note that the scalar function $F(\boldsymbol{\rho})$ determines both components of the vector field $\mathbf{S}(\boldsymbol{\rho})$ reducing by this way the complexity of the problem.

The current density across the Josephson junction $j_{x}(0, y)$ is a sum of two terms, namely, the tunnel and the displacement current densities

$$
j_{x}(0, y)=\left[j_{c} \sin \varphi+\frac{\hbar C}{2 e} \frac{\partial^{2} \varphi}{\partial t^{2}}\right] d \delta(z)
$$

At the same time it follows from Eq. (10) that the current density $j_{x}(0, y)$ can be written as

$$
j_{x}(0, y)=\frac{c}{4 \pi \lambda_{\text {eff }}}\left[S_{x}(0, y)-A_{x}(0, y, 0)\right] \delta(z)
$$

Equating the expressions for the quantity $j_{x}(0, y)$ given by Eqs. (20) and (21) we find that

$$
j_{c} \sin \varphi+\frac{\hbar C}{2 e} \frac{\partial^{2} \varphi}{\partial t^{2}}=\frac{c}{4 \pi \lambda^{2}}\left[S_{x}(0, y)-A_{x}(0, y, 0)\right]
$$

Thus, to derive the closed form of the equation describing the phase difference $\varphi(y, t)$ it is necessary to find the functional relation between

$$
\Delta_{x}(y)=S_{x}(0, y)-A_{x}(0, y, 0)
$$

and $\varphi(y, t)$. We use here Fourier transformation in order to do it and defining the Fourier transforms for $\mathbf{A}(\mathbf{r})$ and $\mathbf{S}(\boldsymbol{\rho})$ as

$$
\mathbf{A}(\mathbf{r})=\int \mathbf{A}_{\mathbf{q}, p} \exp (i \boldsymbol{q} \boldsymbol{\rho}+i p z) \frac{d^{2} \mathbf{q} d p}{(2 \pi)^{3}}
$$

and

$$
\mathbf{S}(\boldsymbol{\rho})=\int \mathbf{S}_{\mathbf{q}} \exp (i \boldsymbol{q} \boldsymbol{\rho}) \frac{d^{2} \mathbf{q}}{(2 \pi)^{2}}
$$

Using $\mathbf{A}_{\mathbf{q}, p}$ and $\mathbf{S}_{\mathbf{q}}$ we can present the value of $\Delta_{x}$ by the integral 


$$
\Delta_{x}=\frac{1}{4 \pi^{2}} \int_{0}^{\infty} q d q \int_{-\pi}^{\pi} d \vartheta\left(S_{\mathbf{q}}^{x}-A_{\mathbf{q}}^{x}\right) \exp (i q y \sin \vartheta)
$$

where $\vartheta$ is the polar angle in the $\left(q_{x}, q_{y}\right)$-plane and

$$
\mathbf{A}_{\mathbf{q}}=\int_{-\infty}^{\infty} \mathbf{A}_{\mathbf{q}, p} \frac{d p}{2 \pi}
$$

The next step is to apply Fourier transformation to Eq. (14), which results in

$$
\left(q^{2}+p^{2}\right) \mathbf{A}_{\mathbf{q}, p}+\lambda_{\text {eff }}^{-1} \mathbf{A}_{\mathbf{q}}=\lambda_{\text {eff }}^{-1} \mathbf{S}_{\mathbf{q}} .
$$

It follows from Eq. (28) that the relation between $\mathbf{A}_{\mathbf{q}}$ and $\mathbf{S}_{\mathbf{q}}$ has the form

$$
\mathbf{A}_{\mathbf{q}}=\frac{\mathbf{S}_{\mathbf{q}}}{1+2 q \lambda_{\text {eff }}}
$$

and thus

$$
S_{\mathbf{q}}^{x}-A_{\mathbf{q}}^{x}=\frac{2 q \lambda_{\mathrm{eff}}}{1+2 q \lambda_{\mathrm{eff}}} S_{\mathbf{q}}^{x} .
$$

To calculate the Fourier transform $S_{\mathbf{q}}^{x}$ we take the derivative of Eq. (19) with respect to $y$ and substitute $S_{x}$ instead of $\partial F / \partial y$. As a result it comes out that

$$
\Delta S_{x}=-\frac{\Phi_{0}}{2 \pi} \varphi^{\prime \prime}(y) \delta(x) .
$$

It follows from Eq. (31) that the Fourier transform $S_{\mathbf{q}}^{x}$ is given by the formula

$$
S_{\mathbf{q}}^{x}=\frac{\Phi_{0}}{2 \pi q^{2}} \int_{-\infty}^{\infty} d y \varphi^{\prime \prime}(y) \exp (-i q y \sin \vartheta)
$$

Combining now Eqs. (32), (30), (26), and (22) we find the integro-differential equation describing the phase difference $\varphi(y, t)$ in the form

$$
\frac{1}{\omega_{J}^{2}} \frac{\partial^{2} \varphi}{\partial t^{2}}+\sin \varphi=l_{J} \int_{-\infty}^{\infty} d y^{\prime} K\left(\frac{y-y^{\prime}}{2 \lambda_{\mathrm{eff}}}\right) \varphi^{\prime \prime}\left(y^{\prime}\right),
$$

where

$$
K(u)=\frac{1}{\pi} \int_{0}^{\infty} \frac{J_{0}(v)}{v+|u|} d v
$$


$J_{0}(v)$ is the zero-order Bessel function, and

$$
l_{J}=\frac{c \Phi_{0}}{16 \pi^{2} \lambda^{2} j_{c}} .
$$

Note that Eq. (33) can be rewritten as

$$
\frac{1}{\omega_{J}^{2}} \frac{\partial^{2} \varphi}{\partial t^{2}}+\sin \varphi=\frac{l_{J}}{\pi} \int_{-\infty}^{\infty} \frac{d y^{\prime}}{y^{\prime}-y} \frac{\partial \varphi}{\partial y^{\prime}}
$$

in the limiting case when the characteristic space scale of the phase difference variation is much less than $\lambda_{\text {eff }}$.

\section{DISPERSION RELATION}

Let us now consider a small amplitude electromagnetic wave propagating along the Josephson junction. The corresponding solution of Eq. (33) then reads

$$
\varphi=\varphi_{0} \exp (i k y-i \omega t), \quad\left|\varphi_{0}\right| \ll 1 .
$$

Substitution of Eq. (37) into Eq. (33) results in the following dispersion relation

$$
\omega=\omega_{J} \sqrt{1+2 k^{2} \lambda_{\mathrm{eff}} l_{J} \mathcal{K}\left(2 k \lambda_{\mathrm{eff}}\right)},
$$

where

$$
\mathcal{K}(x)=\int_{-\infty}^{\infty} K(u) \exp (i x u) d u .
$$

The function $\mathcal{K}(x)$ has the following explicit form

$$
\mathcal{K}(x)= \begin{cases}\frac{1}{\pi \sqrt{1-x^{2}}} \ln \frac{1+\sqrt{1-x^{2}}}{1-\sqrt{1-x^{2}}}, & \text { if } x<1 ; \\ \frac{1}{\sqrt{x^{2}-1}}\left[1-\frac{2}{\pi} \arctan \frac{1}{\sqrt{x^{2}-1}}\right], & \text { if } x>1 .\end{cases}
$$

Using Eqs. (38) and (40) we find, in particular, the dispersion relation $\omega(k)$ in the limiting cases $k \lambda_{\text {eff }} \ll 1$ and $k \lambda_{\text {eff }} \gg 1$ 


$$
\omega= \begin{cases}\omega_{J} \sqrt{1-\frac{4 k^{2} \lambda_{\mathrm{eff}} l_{J}}{\pi} \ln \left(k \lambda_{\mathrm{eff}}\right),} & \text { if } k \lambda_{\mathrm{eff}} \ll 1 ; \\ \omega_{J} \sqrt{1+k l_{J}}, & \text { if } k \lambda_{\mathrm{eff}} \gg 1 .\end{cases}
$$

Thus, for $k \lambda_{\text {eff }} \gg 1$ and $k l_{J} \gg 1$ the frequency of an electromagnetic wave propagating along the SIS-type tunnel Josephson junction in a thin film is proportional to the square root of the wave vector.

The dispersion relation $\omega \propto \sqrt{k}$ leads, in particular, to a non-equidistant set of the self-induced Fiske resonances in the current-voltage curves for the voltages $V>V_{c}$, where

$$
V_{c}=\frac{\hbar \omega\left(\lambda_{\mathrm{eff}}^{-1}\right)}{2 e} \approx \frac{c_{s}}{\lambda} \sqrt{\frac{d}{\lambda}} .
$$

Note, that for a thin superconducting film the relation $k \lambda_{\text {eff }} \sim 1$ corresponds to a wave length that is $\lambda / d$ times bigger than the London penetration depth.

\section{SUMMARY}

To summarize, we have found the integro-differential equation describing the phase difference in case of the SIS-type tunnel Josephson junction in a thin superconducting film. We apply this equation to calculate the dispersion relation for an electromagnetic wave propagating along the Josephson contact. We have shown that if the wave length is small compared with the Pearl penetration depth $\lambda_{\text {eff }}$ the frequency is proportional to the square root of the wave vector.

\section{ACKNOWLEDGMENTS}

We are grateful to Dr. E. Polturak for useful discussions. This work was supported in part by the Foundation Raschi. 


\section{REFERENCES}

${ }^{1}$ A. Barone and G. Paterno, Physics and Applications of the Josephson Effect (Wiley, New York, 1982).

2 J.C. Swihart, J. Appl. Phys. 32, 461 (1961).

${ }^{3}$ M.D. Fiske, Rev. Mod. Phys. 36, 221 (1964).

${ }^{4}$ D. Winkler, Y.M. Zhang, P.Å. Nilsson, E.A. Stepanov and T. Claeson, Phys. Rev. Lett. 72, 1260 (1994).

${ }^{5}$ R.E. Ekk, D.J. Scalapino and B.N. Taylor Phys. Rev. Lett. 13, 15 (1964).

${ }^{6}$ I.O. Kulik, Pis'ma Zh. Eksp. Teor. Fiz. 2, 134 (1965) [JETP Lett. 2, 84 (1965)].

${ }^{7}$ A. Gurevich, Phys. Rev. B 46, 3187 (1992)

${ }^{8}$ R.G. Mints and I.B. Snapiro, Physica A 200, 426 (1993).

${ }^{9}$ R.G. Mints and I.B. Snapiro, Phys. Rev. B 49, 6188 (1994).

${ }^{10}$ R.G. Mints and I.B. Snapiro, submitted to Phys. Rev. B (1994).

${ }^{11}$ J. Pearl, Appl. Phys. Lett. 5, 65 (1964)

${ }_{12}$ M. Tinkham, Introduction to Superconductivity (McGraw Hill, Inc., New York, 1975).

${ }^{13}$ P.G. De Gennes, Superconductivity of Metals and Alloys (W.A. Benjamin, Inc., New York, 1966). 


\section{FIGURES}

FIG. 1. A thin superconducting film with a Josephson junction (thick line). 